\title{
COMPARACIÓN DE LAS COMUNIDADES DE MACROINVERTEBRADOS ACUÁTICOS EN RÍOS INTERMITENTES Y PERMANENTES DEL ALTIPLANO BOLIVIANO: IMPLICACIONES PARA EL FUTURO CAMBIO CLIMÁTICO
}

\section{COMPARISON OF AQUATIC MACROINVERTEBRATE COMMUNITIES FROM INTERMITTENT AND PERENNIAL BOLIVIAN HEADWATER ANDEAN STREAMS: IMPLICATIONS FOR FUTURE CLIMATE CHANGE}

\author{
Nabor Moya ${ }^{1,2}$, François-Marie Gibon $^{3,4}$, Thierry Oberdorff ${ }^{2}$, Claudio Rosales $^{4}$ y Eduardo Domínguez ${ }^{5}$
}

\section{Resumen}

El impacto de la estacionalidad de los ríos sobre las comunidades de macroinvertebrados fue estudiado en ríos de la zona Altoandina de Bolivia, durante la época de transición y época seca del año 2008. En nueve arroyos intermitentes y nueve permanentes se colectaron muestras de macroinvertebrados y parámetros ambientales. A traves de análisis multivariados, se determinaron relaciones entre variables biológicas (según riqueza, abundancia, composición trófica y rasgos biológicos) con las variables ambientales. La estacionalidad no tuvo efecto significativo sobre la riqueza ni densidad de macroinvertebrados, tampoco se encontraron efectos notorios sobre la composición trófica ni los rasgos biológicos (tipo de respiración), pero sí encontramos diferencias significativas en la composición taxonómica, especialmente en la proporción de Ephemeroptera, Plecoptera y Trichoptera (EPT); asimismo, encontramos que existen otras variables ambientales como la profundidad, solidos disueltos y $\mathrm{pH}$ que tienen mayor efecto que la intermitencia en la riqueza y densidad total. De acuerdo a datos climáticos de la región Andina de Bolivia se sabe que en el futuro habrá una disminución del agua, incluso la desecación temporal en varios ríos de la zona, esto debido a la disminución de los glaciares. De esta manera, el futuro cambio climático reflejado a través de la estacionalidad de los ríos, tendrá mayor efecto en los cambios de la estructura taxonómica que en la estructura funcional, lo que indica que probablemente tendrá implicaciones más fuertes en la conservación de taxa que en la composición de los rasgos de la comunidad de macroinvertebrados.

Palabras clave: insectos acuáticos, estacionalidad de ríos, glaciares andinos, Bolivia

\begin{abstract}
The impact of hydrologic non-permanence (intermittent streams) on benthic macroinvertebrate communities was investigated in Bolivian head Andean streams. Samples of benthic macroinvertebrates and environmental variables were taken in nine intermittent and nine permanent streams during dry and transition to dry seasons during 2008. Using different multivariate analyses, we determined relationships between biological variables (richness, density, trophic composition and biological traits) and environmental variables. Hydrologic nonpermanence had no significant effect on total species richness, total density, trophic composition or physiological traits (i.e. respiration). However, significant effects were found on taxonomic composition, specifically on taxonomic richness of Ephemeroptera, Plecoptera and Trichoptera. Likewise, we found that environmental variables such as profundity, total dissolved solids and $\mathrm{pH}$ have more important effects on total richness and density than hydrologic non-permanence itself. Thus, climate change would likely have stronger implications for taxa conservation than for the functional characteristics of stream macroinvertebrate communities.
\end{abstract}

Key words: aquatic insects, intermittent streams, Andean glaciers, Bolivia

\section{Introducción.}

El segundo grupo de trabajo del IPCC (2007) concluye que una evaluación global que data desde 1970 muestra que el calentamiento global antropogénico tiene probablemente entre el 66 a 90\% de probabilidad de ocurrencia, lo cual tiene una influencia discernible sobre muchos sistemas biológicos y físicos. Cerca del $90 \%$ de los glaciares andinos se encuentran en zonas con pronunciadas sequías y el $10 \%$ en regiones húmedas tropicales. El cambio climático se pronuncia en las regiones más secas, afectando la distribución anual del escurrimiento con diferencias marcadas entre la estación seca $\mathrm{y}$ húmeda $\mathrm{e}$ incrementando la variabilidad interanual (InWEnt, 2008).

Los glaciares, especialmente los tropicales, son 


\section{MACROINVERTEBRADOS ACUÁTICOS EN RÍOS INTERMITENTES Y PERMANENTES}

Julio - Diciembre 2009

excelentes indicadores de la evolución del clima; son ecosistemas vulnerables y constituyen las reservas sólidas de agua dulce. Este recurso es utilizado en el consumo, agricultura, hidroelectricidad, actividad minera y proyectos agroindustriales, juega un rol importante en el desarrollo socioeconómico de las poblaciones y una reducción en su disponibilidad podría generar una crisis en los suministros y calidad (InWEnt, 2008). Actualmente, de acuerdo a datos climáticos de la región Andina de Bolivia (Vuille et al., 2008; Francou \& Pouyaud, 2008), se sabe que existe una disminución e incluso una desecación temporal de varios ríos de la zona, esto debido a la disminución de los glaciares de la zona, como por ejemplo el Nevado Sajama.

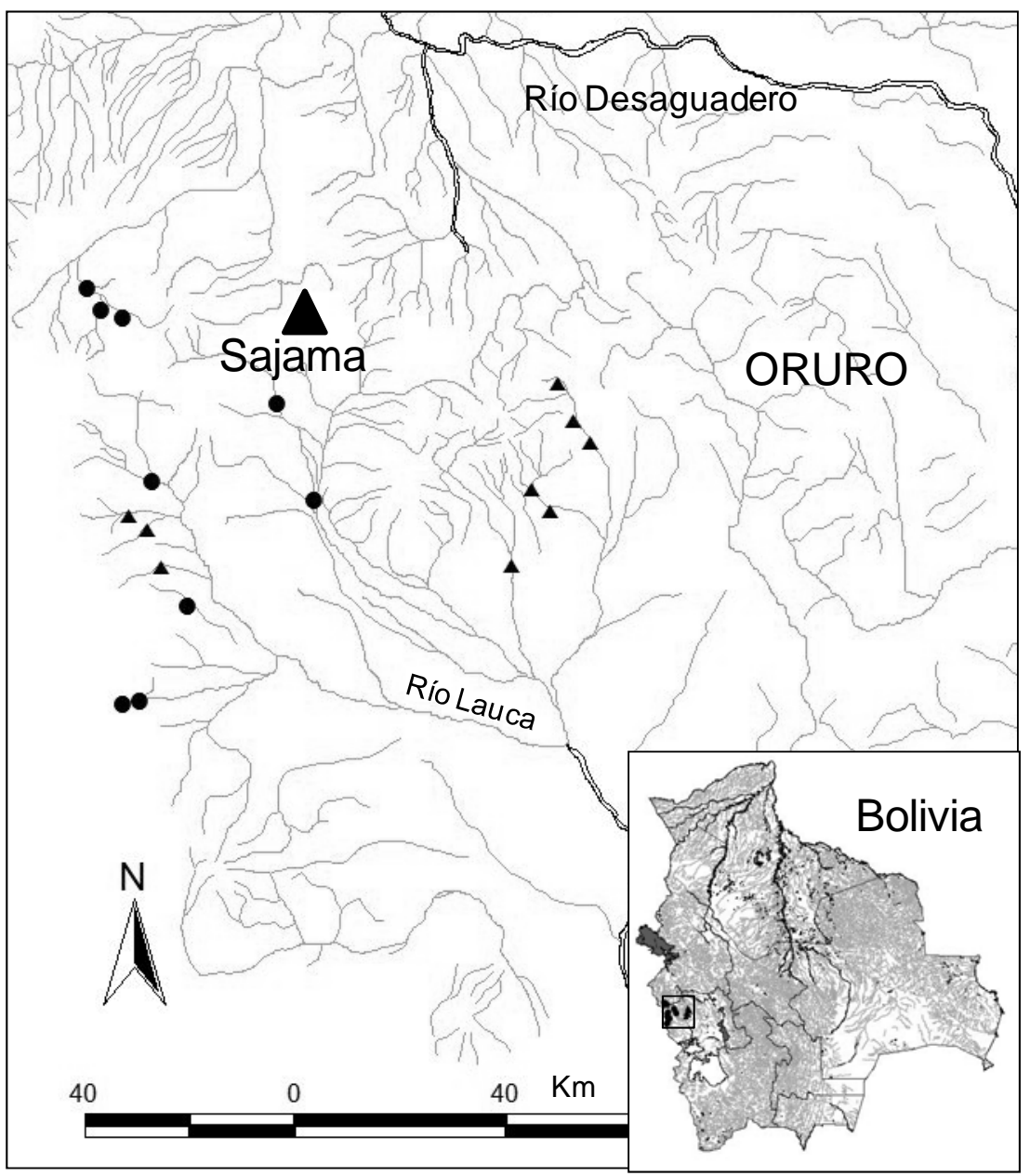

Figura 1. Ubicación de la zona de estudio en el Departamento de Oruro, Bolivia. Los círculos negros corresponden a sitios permanentes y los triángulos a los sitios intermitentes.

El uso de los macroinvertebrados acuáticos tienen interés particular, debido a las grandes ventajas que presentan estas comunidades en la evaluación y monitoreo de ambientes acuáticos (p.e. Bonada et al., 2006a). Entre las principales ventajas se tiene: la facilidad de muestreo, el rápido cambio en la estructura trófica, composición y abundancia de algunos grupos a diversos tipos de perturbación natural y antropogénica y su naturaleza sedentaria que proporciona una buena señal espacial de lo que ocurre en cada hábitat muestreado (Bailey et al., 2003). Asimismo, se sabe que estas comunidades son sensibles a pequeños incrementos de temperatura (Bergkamp \& Orlando, 1999; Durance \& Ormerod 2007), por lo cual los efectos del calentamiento global son de suma relevancia.

Existe abundancia de datos empíricos que sugieren que el reciente cambio climático induce variaciones estructurales (p.e. perdida de especies, cambios en la distribución de las especies; Daufresne \& Boët, 2007), y funcionales (p.e. fenología, migración, tamaño de cuerpo; Bonada et al. 2007).

Muchos de estos estudios, sin embargo, son enfocados a escalas locales y en especies simples. La extrapolación desde niveles de organización bajo (especie) a niveles altos (comunidad) es difícil, y el cambio climático es típicamente visto como un fenómeno a escala grande.

Por ello, estudios a escalas espaciales grandes y niveles de organizaciones biológicas altas, son requeridos a anticipar impactos del cambio climático. Bajo ese contexto, Bonada et al. (2007) estudiaron las diferencias en rasgos biológicos y composición taxonómica de macroinvertebrados entre regiones mediterráneas y templadas que experimentan periodos secos, concluyendo que tanto la diversidad $y$ riqueza de rasgos biológicos fueron más altos en regiones mediterráneas. Asimismo, tanto la composición taxonómica $\mathrm{y}$ los rasgos biológicos difirieron entre las dos regiones.

Bajo esa misma línea, Durance \& Ormerod (2007), estudiaron los efectos del cambio climático sobre la fauna de macroinvertebrados en ríos de tierras altas del NorAtlántico por un periodo de 25 años. Ellos sostienen que la abundancia de macroinvertebrados podría disminuir en un $21 \%$ por cada incremento de $1^{\circ} \mathrm{C}$. Si bien muchas especies resistentes podrían persistir si la temperatura aumenta en $3^{\circ} \mathrm{C}$, entre el 5 y $12 \%$ de las especies más raras estarían en riesgo de extinción local. 
En Sudamérica, si bien existe un creciente número de trabajos sobre las comunidades de macroinvertebrados acuáticos, especialmente como bioindicadoras en evaluaciones de la calidad de ríos (Figueroa et al., 2003; Silveira et al., 2005; Couceiro et al., 2007; Baptista et al., 2007; Moya et al., 2007), sin embrago ninguno de ellos toman en cuenta en su procedimiento los patrones de variación bajo las implicancias del cambio climático.

Bajo ese contexto es que este trabajo, pretende estudiar y discutir los patrones de variación de las comunidades de macroinvertebrados acuáticos bajo las implicancias del cambio climático (reflejado a través de la estacionalidad de los ríos) haciendo una comparación de estas comunidades en ríos intermitentes y permanentes del altiplano Boliviano. $\underline{\text { Área de estudio }}$

La zona de estudio pertenece a parte de los departamentos de Oruro y La Paz de Bolivia, corresponde a la ecoregión Altoandina de Bolivia (Figura 1). El bioclima es predominantemente xérico, con una precipitación media anual de $193 \mathrm{~mm}$ y con una temperatura media de $5.5^{\circ} \mathrm{C}$ (Navarro \& Maldonado, 2002). La altitud varía desde los 3800 hasta los $6500 \mathrm{~m}$. Esta zona incluye al Nevado Sajama, que es uno de los picos más altos de Bolivia.

Inicialmente, según mapas del IGM y visitas previas a la zona de estudio, se seleccionaron 18 sitios, los cuales se dividieron en dos grupos; un grupo de 9 sitios con aguas permanentes durante todo el año y el otro grupo de 9 sitios con aguas estacionales o intermitentes al menos durante dos meses secos.

\section{Materiales y métodos.}

Se tomaron muestras de macroinvertebrados bentónicos y medidas ambientales en los 18 sitios durante la época seca (julio-octubre) del año 2008, siguiendo el protocolo de Karr \& Chu (1999). En aquellos ríos estacionales, se colectaron antes de su desecación total (abril-junio).

En cada sitio de muestreo, se tomaron cinco muestras Surber, cada una de $0.1 \mathrm{~m}^{2}$ fueron colectados en los segmentos de rabión más naturales; en lo posible cada uno de los puntos correspondió a un simple rabión (rápido) con hábitats similares, según el protocolo de Karr \& Chu (1999). Las cinco muestras Surber en conjunto se conservaron en frascos de 0.51 con formol al $4 \%$. Una vez en el laboratorio, los macroinvertebrados fueron separados e identificados hasta el nivel taxonómico posible, usando claves de Fernández \& Domínguez (2001) y Merrit \& Cummins (1996). Con lo que se determinaron variables biológicas según riqueza, abundancia, composición y estructura trófica, tal como se describe en Moya et al. (2007)

Así mismo, en cada estación se tomaron medidas ambientales como la altitud, el ancho activo y total, la profundidad, velocidad de corriente, longitud rabión, frecuencia de cada tipo de substrato, según la escala granulométrica de Wenthworth (entre 80 a 100 medidas, cada una separadas por $1 \mathrm{~m}$ en zigzag a lo largo del tramo.

La inestabilidad de substrato, a través de observaciones visuales se dividió en 4 categorías, donde: $0=$ estable (sustrato predominantemente grueso, con desarrollo de algas), 1= algo estable, $2=$ inestable, $3=$ muy inestable (sustrato predominantemente fino $y$ movilizado por la corriente).

Para incluir a los análisis estadísticos, las clases de tamaño del substrato fueron categorizados como proponen Heino et al. (2003) con ligeras modificaciones: (1) materia orgánica, limo, arcilla; (2) arena, (3) grava fina, (4) grava gruesa, (5) cascajo fino, (6) cascajo grueso, (7) piedra fina, (8) piedra grande, (9) bloque. La proporción promedio de cada tipo de substrato fue estimada y subsecuentemente promediada para dar el valor promedio para cada sitio como un valor de índice

Asimismo, se tomaron medidas químicas como la conductividad, salinidad, oxígeno disuelto, sólidos totales disueltos, temperatura y $\mathrm{pH}$, que son de importancia en la distribución de la fauna bentónica (Vinson \& Hawkins, 1998). Asimismo adicionamos la intermitencia $\mathrm{o}$ estacionalidad como variable categórica, donde $1=$ intermitente y $0=$ permanente.

Las comunidades también se diferenciaron según $\mathrm{su}$ estructura trófica como desmenuzadores, raspadores, colectores, filtradores y predadores $\mathrm{y}$ cuatro rasgos de vida según el tipo de respiración (ya sea por tegumento, branquias, plastrón o estigmas) de acuerdo a códigos compuestos por afinidad (donde $0=\sin$ afinidad del taxón con la categoría de rasgo, $1=$ afinidad débil, $2=$ afinidad media, $3=$ afinidad alta) tal como describe Tomanova et al. (2008) en Bolivia y literatura accesible como Merrit \& Cummins (1996). Análisis de datos

Las diferencias en abundancia de los diferentes taxa, así como de los datos ambientales entre sitios permanentes e intermitentes, se probaron con el test no paramétrico de Mann-Whitney, con un nivel de significancia de 0.10 .

Para tener una visión general acerca de la variabilidad ambiental de la zona estudiada, inicialmente se realizó un Análisis de Componentes Principales (ACP), de tal manera que se visualizó la distribución de los sitios (ríos) tanto permanentes como estacionales, tomando en cuenta solamente las características ambientales no correlacionadas entre ellas y sin incluir la intermitencia.

Para determinar asociaciones entre la estructura de las comunidades (excluyendo los taxa raros $\leq 0.1 \%$ respecto a la abundancia total de los 18 sitios) y las variables ambientales se realizó un Análisis de Correspondencia Canónica (ACC).

El ACC sirve para entender como diversos taxa 


\section{MACROINVERTEBRADOS ACUÁTICOS EN RÍOS INTERMITENTES Y PERMANENTES}

Julio - Diciembre 2009

responden simultáneamente a factores externos como las variables ambientales. El resultado del ACC es un diagrama de ordenamiento formado por un sistema de ejes donde se muestran los sitios, las especies y variables ambientales.

A través de este ACC se espera que la distribución de las especies esté asociada a las variables ambientales; asimismo se espera que los ríos permanentes y estacionales tiendan a diferenciarse en relación a este conjunto de variables.

Asimismo, se realizó un análisis de componentes principales para determinar si hay una asociación entre la proporción de la composición trófica con los ríos, así como con la proporción de los organismos según el tipo de respiración que podrían estar asociados a distintos sitios, ya sea permanente o estacionales.

Para cada una de las variables biológicas, se desarrollaron modelos de regresión stepwise (GLM) utilizando estas variables biológicas como variables dependientes en el modelo, y las variables ambientales como variables independientes, incluida la intermitencia como variable categórica.

Los análisis (ACP y ACC) se realizaron en el programa CANOCO v 4.5, los modelos de regresión y el test de Mann-Whitney con el programa SYSTAT 11.0 .

\section{Resultados y discusión.}

Un total de 37 taxa de macroinvertebrados fueron colectados en los 18 sitios de la zona estudiada, de los cuales 31 corresponden a la clase Insecta y 6 a otras clases (Tabla1)

Una densidad media de $6449( \pm 4248)$ ind $/ \mathrm{m}^{2}$ fueron encontrados en cada río permanente; siendo la familia Chironomidae la más abundante, que representa a un $32 \%$ de abundancia total de los 9 ríos permanentes. Para los ríos estacionales encontramos un total de $4674( \pm 3590) \mathrm{ind} / \mathrm{m}^{2}$, siendo igualmente Chironomidae la más abundante, con un $30 \%$. Un promedio de $15( \pm 2.9)$ taxa fueron encontrados en cada río permanente y un promedio de $14( \pm 3.7)$ taxa en cada río estacional.

Los tricópteros Hydroptilidae e Hydrobiosidae tuvieron mayor densidad en los sitios permanentes que en los intermitentes (test de Mann-Whitney, $\mathrm{p}<0.10$ ), por otro lado Elmidae (adulto), Dytiscidae (adulto) y Ceratopogonidae se presentaron con mayor abundancia en los sitios intermitentes que permanentes (test de Mann-Whitney, $\mathrm{p}<0.10$ ) (Tabla $1)$, el resto de los taxa no presentaron diferencias significativas.

Con respecto a las características funcionales tróficas de la comunidad, los recolectores son los más abundantes con un promedio de 39 y $38 \%$ para los sitios permanentes e intermitentes respectivamente y los predadores los menos abundantes con solo el $10 \mathrm{y}$ $9 \%$ para los sitios permanentes e intermitentes respectivamente. Respecto al tipo de respiración, aquellos que tienen respiración por tegumento fueron los más abundantes $(55$ y $42 \%$ para sitios permanentes e intermitentes respectivamente) y los que tienen respiración por estigmas fueron los menos abundantes $(6$ y $3 \%$ para sitios permanentes e intermitentes respectivamente).

De forma general, los datos ambientales entre sitios permanentes e intermitentes son similares excepto para el $\mathrm{pH}$ y la profundidad, cuyos valores son superiores en los sitios permanentes $(p<0.05)$ (Tabla 2).

El ACP indica que la variación entre las variables ambientales puede ser explicado por los dos primeros componentes principales en un 53\% (Figura 2). El primer eje separa los sitios de acuerdo a variables físicas como el tipo de substrato, la estabilidad de substrato y la altitud. El segundo eje ordena los sitios en función a las variables químicas como la conductividad, sólidos totales disueltos, además de la profundidad, velocidad de corriente y el ancho del río. Cabe aclarar que los puntos 106 y 95 tienen valores extremos de conductividad y STD, probablemente debido a condiciones geológicas y de relieve particulares que permiten mayor concentración de sales, aunque en general toda la zona de estudio es de alta salinidad, pues casi todos los ríos estudiados descargan en el Salar de Coipasa.

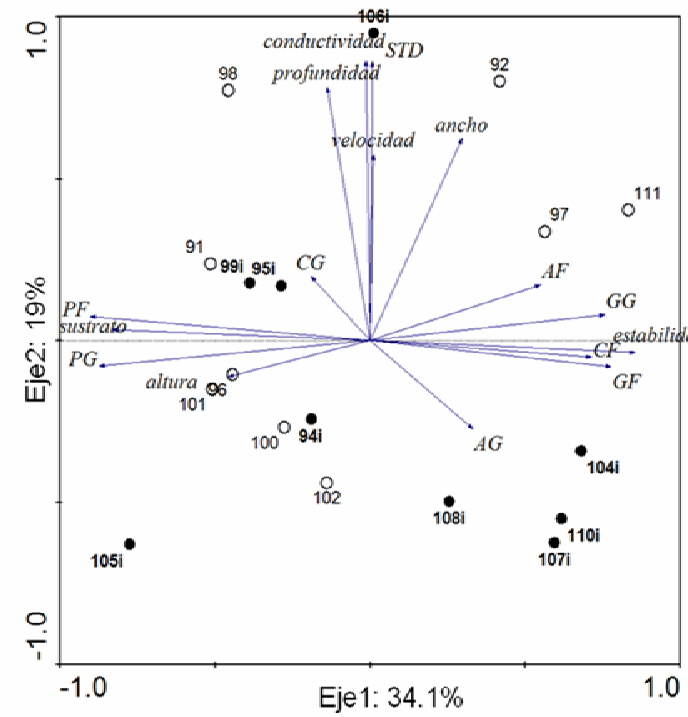

Figura 2. Ordenamiento de los sitios según el ACP de acuerdo a variables ambientales. Los puntos negros corresponden a los sitios intermitentes. $\mathrm{STD}=$ sólidos totales disueltos, $\mathrm{PF}=$ piedra fina, $\mathrm{PG}=$ piedra gruesa, $\mathrm{AF}=$ arena fina, $\mathrm{AG}=$ arena gruesa, $\mathrm{GF}=$ grava fina, $\mathrm{GG}=$ grava gruesa, $\mathrm{CF}=$ cascajo fino, $\mathrm{CG}=$ cascajo grueso.

Con este análisis se muestra que los sitios intermitentes se encuentran distribuidos dentro de la variabilidad ambiental de los sitios permanentes, 
debido a que ambos grupos de sitios se encuentran dispersos en este diagrama de ordenación (Figura 2), es decir, que los sitios intermitentes comparten características ambientales similares a los permanentes y por tanto no habrá problema en aplicar para los posteriores análisis en ambos grupos de sitios, tanto permanentes e intermitentes.

El Análisis de Correspondencia Canónica (ACC) (Figura 3), indica que la varianza acumulada de la relación entre las variables ambientales y biológicas puede ser explicada por los dos primeros ejes en un $38 \%$.

En la figura 3 (a y b) se observa que los sitios intermitentes (códigos rojos) tienen una tendencia de separación de los sitios permanentes hacia la parte inferior del eje 2 (ACC2).

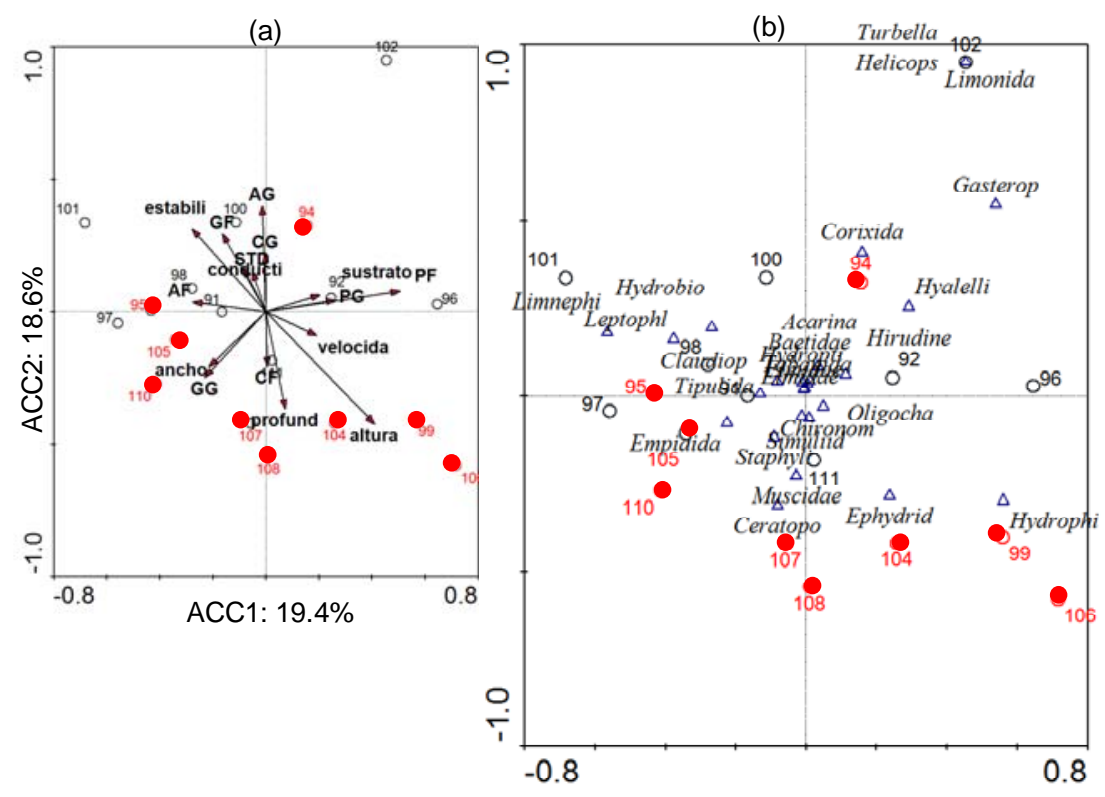

Figura 3. Resultado del Análisis de Correspondencia Canónica (ACC) en el ordenamiento de las estaciones muestreadas en la zona estudiada, según la abundancia de la comunidad de macroinvertebrados (transformado con $\ln +1$ ) (b) relacionado con las variables ambientales (a). Los códigos rojos corresponden a los sitios intermitentes, los negros a los sitios permanentes, las flechas indican las variables ambientales y los triángulos los taxa. Para códigos véase figura 2.

En aquellos sitios estacionales (parte inferior del diagrama del ACC, en rojo) con valores elevados de altitud, profundidad, ancho y dominancia de cascajo fino (CF) y grava gruesa (GG) predominan los dípteros (Ceratopogonidae, Ephydridae, Muscidae, Empididae, Chironomidae, entre otros (Figura 3 a y b), que tienen mayores facilidades de adaptación y por lo tanto son los más tolerantes a efectos de condiciones extremas o de perturbación (Metcalfe, 1994; DeShon, 1995).

En el otro lado (parte superior de la Figura 3 a y b, en negro), correspondiente a los sitios permanentes, son los grupos sensibles o intolerantes como los tricópteros y efemerópteros (Helicopsychidae, Limnephilidae, Hydrobiosidae, Leptophlebiidae, entre otros) (Metcalfe, 1994; DeShon, 1995; Karr \& Chu,1999; Butcher et al, 2003) los que tienden a predominar; cuyos valores de las variables ambientales anteriormente mencionadas tienden a ser más bajos, pero con dominancia en general de sustratos pedregosos y de mayor estabilidad.

De forma general, esta Figura 3 muestra una tendencia de separación entre sitios permanentes e intermitentes en función a la estructura de comunidades bentónicas y las características ambientales.

La diferencia entre los dos tipos de sitios podría estar relacionada con el ciclo de vida más corto de los taxa encontrados en ríos intermitentes (rasgo biológico no probado en este trabajo). La mayoría de las familias de Diptera, generalmente de crecimiento rápido $\mathrm{y}$ tolerantes a la disminución de la calidad del agua, son las que encontramos más frecuentes en los ríos intermitentes (Tabla 1). Así como también, la de otros órdenes de tamaño pequeño (Hydroptilidae) o muy adaptadas a disminuciones de corriente (Baetidae). Estas características las hacen adecuadas para resistir disminuciones del flujo de corriente propias de ríos intermitentes. Familias con requerimientos más altos y de mayor tamaño como Leptophlebiidae e Hydrobiosidae no podrían prosperar en estos ríos.

Al comparar sitios permanentes e intermitentes en ríos de Estados Unidos y Europa Bonada et al. (2006b, 2007) encontraron distintos resultados a este trabajo, donde la composición taxonómica de ríos permanentes estuvo caracterizado básicamente por dominancia de efemerópteros, plecópteros y tricópteros, en cambio ríos estacionales fueron caracterizados por odonatos, coleópteros y heterópteros. En nuestro trabajo, encontramos que tanto sitios permanentes e intermitentes fueron básicamente dominados por dípteros (Chironomidae y Simuliidae) y coleópteros (Elmidae) (Tabla 1). Sólo una familia de Ephemeroptera (Baetidae) y dos de Trichoptera (Hydroptilidae e Hydrobiosidae) tienen abundancias relativamente más altas en sitios permanentes que en intermitentes, 


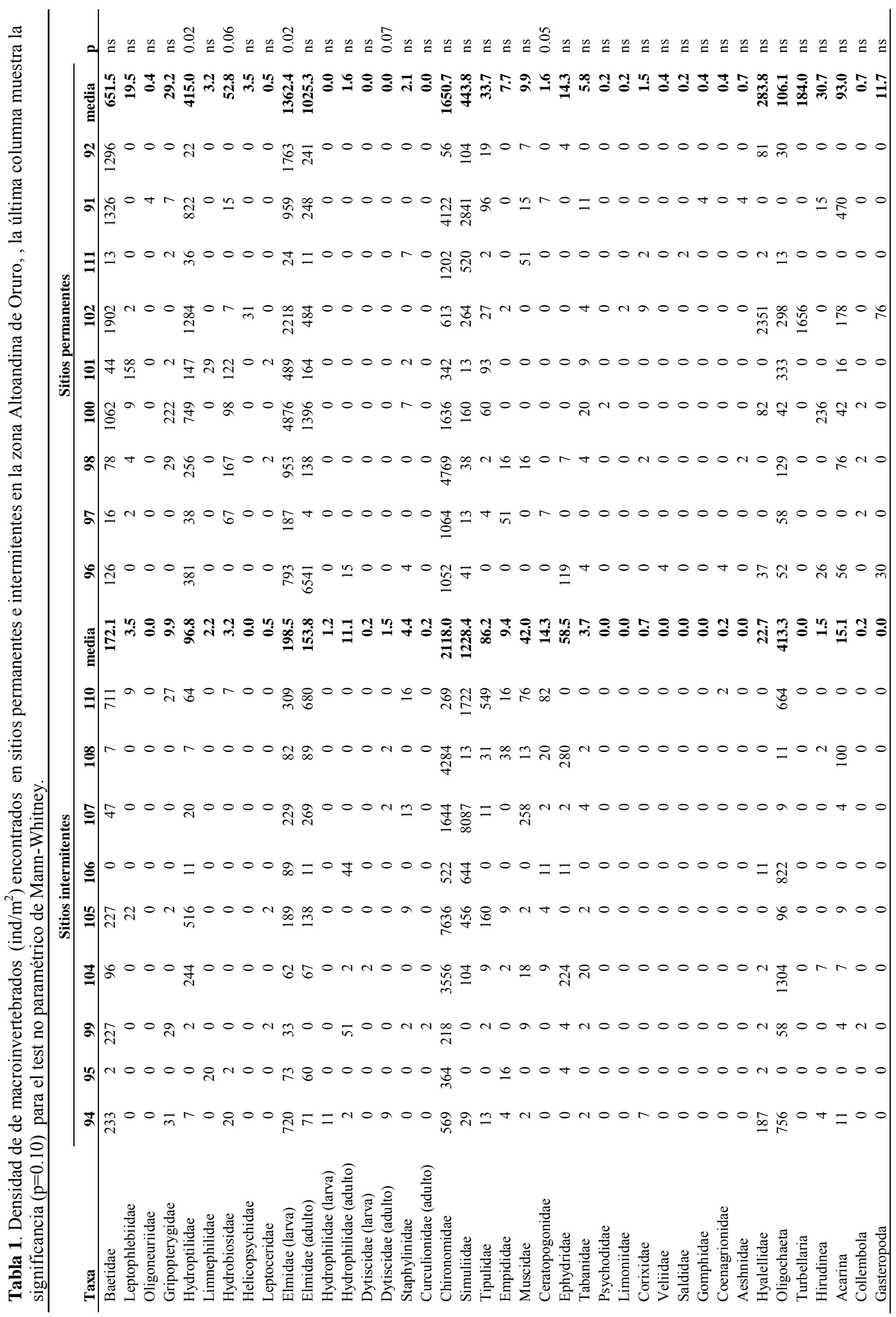




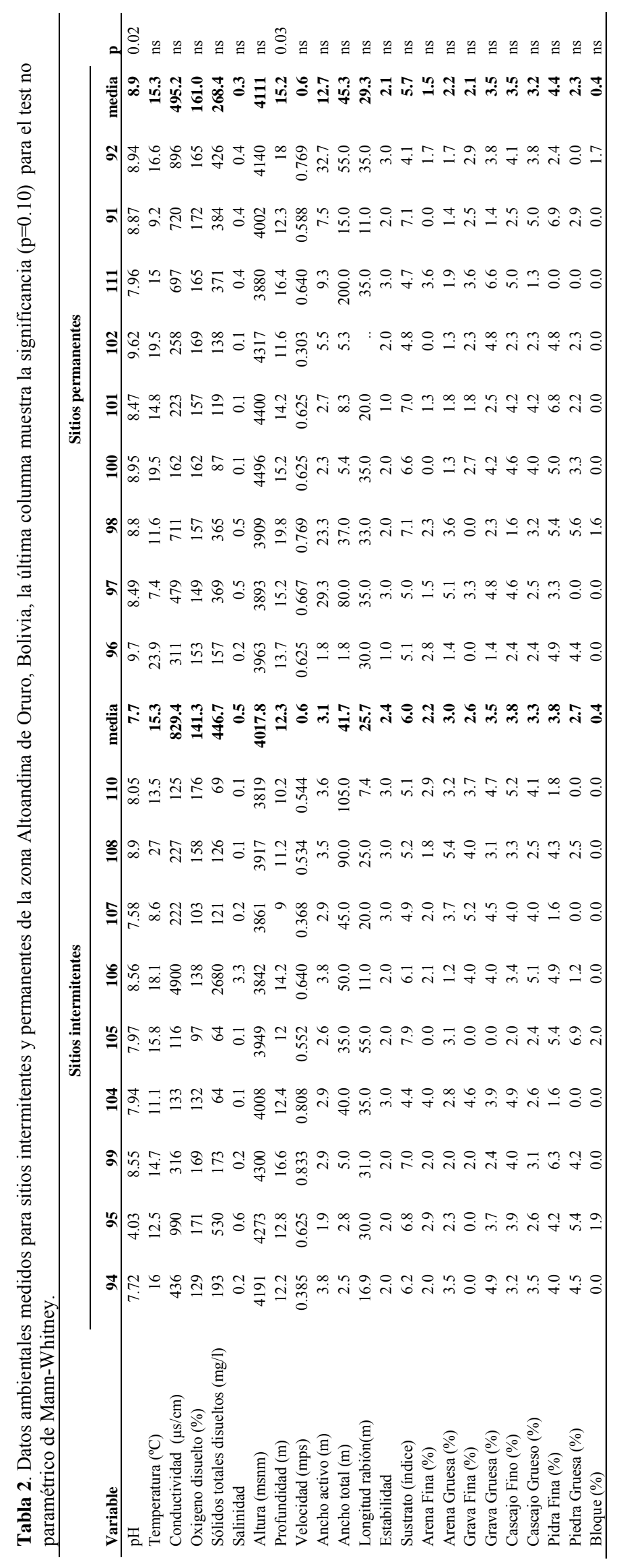


El análisis de componentes principales según la abundancia relativa de la composición trófica de macroinvertebrados bentónicos, indica que esta variabilidad es explicada en un $84.1 \%$ por los dos primeros ejes (Figura 4).

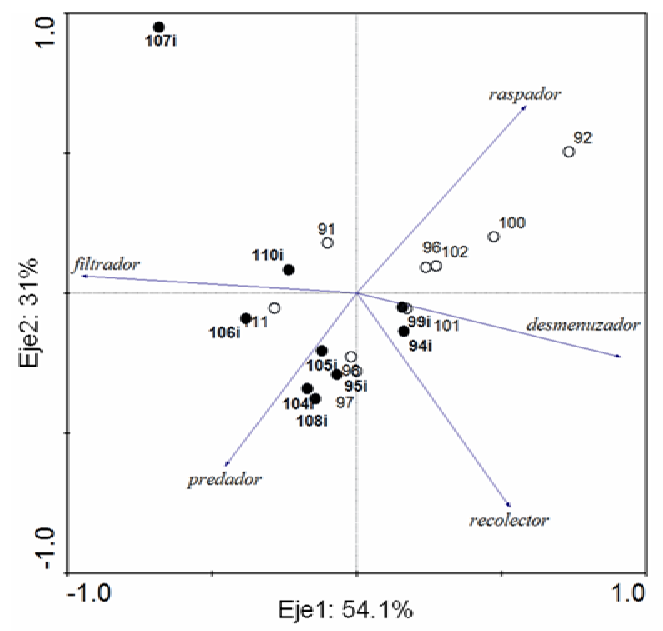

Figura 4. Ordenación de los ríos estudiados según el ACP de acuerdo a la abundancia relativa de la composición trófica de macroinvertebrados, con transformación $\ln +1$. Los puntos negros indican los sitios intermitentes.

Este diagrama indica que hay una tendencia relativa de separación de los sitios intermitentes (en negro) hacia el lado izquierdo, caracterizados por mayor proporción de filtradores y predadores; en cambio los sitios permanentes que tienden a separarse al lado derecho del diagrama, se caracterizan por una dominancia relativa de raspadores $\mathrm{y}$ en parte por recolectores y desmenuzadores.

Sin embargo esta diferenciación entre sitios permanentes e intermitentes no es muy notoria, lo cual indica que los macroinvertebrados tienen capacidades de adaptación a distintos ítems alimenticios, independientemente de la estacionalidad de los ríos, lo que les da mayores ventajas de subsistencia, tal como sostienen Tomanova et al. (2008).

El ACP según el tipo de respiración de macroinvertebrados, muestra que la variabilidad de las categorías de estos rasgos biológicos puede ser explicada en un $80.6 \%$ por los dos primeros ejes (Figura 5).

De forma general, no se aprecian claras diferencias de separación entre sitios intermitentes y permanentes según el tipo de respiración, lo cual indica que los macroinvertebrados estudiados, igual que en el caso de la composición trófica comparten rasgos similares independientemente de la estacionalidad de los ríos. Sin embargo se aprecia una ligera separación de dos sitios permanentes (92 y 96) que tienen organismos que respiran básicamente por

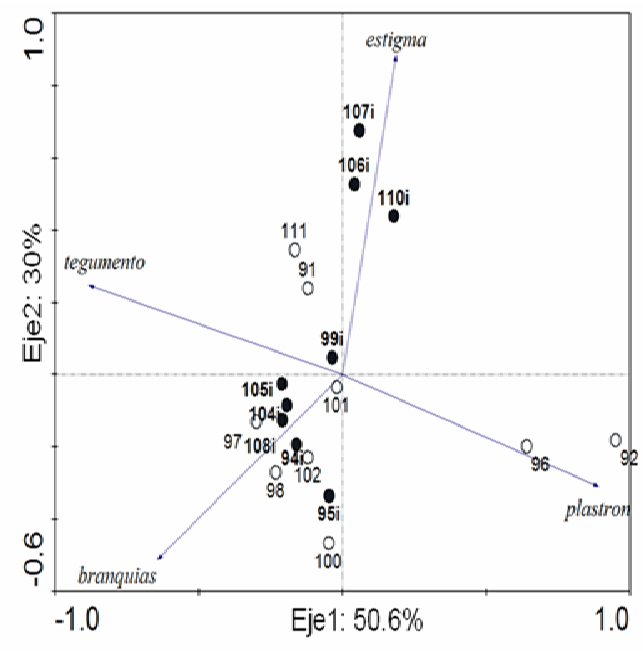

Figura 5. Ordenación de los ríos estudiados según el ACP de acuerdo a categorías de rasgos (respiración) de macroinvertebrados, con transformación $\ln +1$. Los puntos negros indican los sitios intermitentes.

plastrón, y los sitios intermitentes (106, 107 y 110) con organismos de respiración por estigmas.

Otro factor importante a considerar es la dificultad de recolonización. A elevadas altitudes, los vientos hacen el vuelo de los adultos más difícil, es el caso de algunas especies que tienen alas reducidas o atrofiadas, como las hembras de Cailloma rubemarini (Trichoptera, Hydrobiosidae) y los machos de Claudioperla sp. (Plecoptera, Gripopterygidae) (Molina \& Gibon, 2009).

La tabla 3 muestra los modelos de las tres variables biológicas analizadas con el análisis de regresión múltiple, en los tres modelos, los coeficientes $\mathrm{R}^{2}$ son superiores a 0.70 con $\mathrm{p}<0.01$.

De acuerdo a estos modelos, bajo condiciones naturales, hay distintos factores ambientales que

Tabla 3. Modelos de regresión múltiple stepwise para la riqueza y abundancia total y la riqueza de Ephemeroptera, Plecoptera y Trichoptera (EPT), en relación a distintas variables ambientales, incluida la intermitencia; $\mathrm{p}<0.01$ en los tres modelos y las seis variables ambientales.

\begin{tabular}{lccc}
\hline Variable & riqueza & abundancia & riqueza \\
\cline { 2 - 4 } & Total & total & EPT \\
\hline Intercepto & 2.764 & 6.183 & - \\
intermitencia & - & - & 0.807 \\
STD & -0.160 & - & - \\
pH & 0.091 & 0.513 & - \\
profundidad & - & -0.157 & - \\
Índice_sustrato & - & - & 0.925 \\
conductividad & - & - & -0.786 \\
\multicolumn{1}{r}{$\mathrm{R}^{2}$} & 0.79 & 0.71 & 0.84 \\
\hline
\end{tabular}


afectan la riqueza y abundancia de organismos, asi como la riqueza EPT.

En este trabajo, la estacionalidad o intermitencia no tiene efecto significativo sobre la riqueza $y$ abundancia total, sin embargo si tiene efecto significativo $(\mathrm{p}<0.01)$ sobre la riqueza de EPT, además del índice de sustrato y la conductividad. Es decir que la riqueza EPT es afectada positivamente tanto por la intermitencia como por el sustrato y de forma negativa por la conductividad.

De acuerdo a nuestros resultados, al menos parte de la variabilidad observada en la composición de la fauna se debe a la intermitencia de los ríos. Sin embargo el área de trabajo que se estudió es relativamente pequeña $\left(4800 \mathrm{~km}^{2}\right)$, además que solo se consideró dos épocas de un año (seca y transición a la seca), de manera que efectos más notorios se apreciarán a escala macroecológica y por décadas de seguimiento (Bonada et al., 2007; Durance \& Ormerod, 2007).

Implicaciones del cambio climático en las comunidades acuáticas

De acuerdo a los cambios climáticos proyectados por el IPCC (2007) para el 2050 y 2080 y los modelos climáticos simulados para los Andes Tropicales (Vuille et al., 2008), indican que los glaciares continuaran en retroceso, muchos de los glaciares pequeños y de poco volumen, ya están en desequilibrio bajo el clima actual y podrían desaparecer en pocas décadas, tal como ya sucedió con algunas. Incluso en aquellas cuencas donde los glaciares no desaparezcan por completo, el cambio en la estacionalidad de los ríos que es amortiguado por los glaciares, afectará significativamente la disponibilidad de agua en los ríos y por tanto sobre la comunidad biótica.

Bajo este escenario es que planteamos hipotéticamente que la intermitencia de los ríos de la zona Altoandina de Oruro, se debe principalmente a la disminución de los glaciares, y que en las próximas décadas, aquellos ríos que aun son permanentes se harán intermitentes, así como los arroyos intermitentes de hoy solo tendrán agua durante los pocos meses de lluvia de verano.

A pesar de que hay cada vez mayor evidencia del cambio climático ocasionado por actividades antropogénicas, predecir con precisión cuál será el efecto del cambio climático sobre la biota es prácticamente imposible, debido a que el ecosistema es demasiado complejo, donde las interacciones entre especies y el mundo físico son muchas, y especies de un mismo nivel trófico compiten y colaboran entre ellas de maneras difíciles de predecir. Además la respuesta de especies acuáticas al cambio climático es diferente a las especies terrestres.

Respondiendo al objetivo de este estudio, el cambio climático reflejado a través de la estacionalidad de los ríos, tiene mayor efecto en los cambios de la estructura taxonómica que en los cambios de la estructura funcional, similar comportamiento encontraron Bonada et al. (2007) para ríos de zonas mediterráneas $\mathrm{y}$ templadas de Europa. Esto significa que en el futuro, el cambio climático probablemente podría tener implicaciones más fuertes en la conservación de taxa que en la composición de los rasgos de la comunidad de macroinvertebrados, pese a que en general la zona del Altiplano tiene naturalmente baja riqueza de macroinvertebrados comparado con otras regiones.

\section{Agradecimientos.}

Al Institut de Recherche pour le Developpement (UMR BOREA), Unidad de Limnología y Recursos Acuáticos de la Universidad Mayor de San Simón y al Proyecto ToxBol por el financiamiento de este trabajo.

\section{Literatura citada.}

Bailey R.C., Norris R.H. \& Reynoldson T.B. 2003. Bioassesment of freshwater ecosystems using the reference condition approach. Springer Press, U.S.A.

Baptista D.F., Buss D.F., Egler M., Giovanelli A., Silveira M.P \& Nessimian J.L. 2007. A multimetric index based on benthic macroinvertebrates for evaluation of Atlantic forest streams at Rio de Janeiro State, Brazil. Hydrobiologia. 575: 83-94.

Bergkamp G. \& Orlando B. 1999. Exploring Collaboration between the Convention on Wetlands (Ramsar, Iran 1971) and the UN Framework Convention on Climate Change. Climate Iniatiative, IUCN, Washington, USA.

Bonada N., Prat N., Resh V.H. \& Statzner B. 2006a. Developments in aquatic insect biomonitoring: a comparative analysis of recent approaches. Annual Review of Entomology. 51: 495-523.

Bonada N., Rieradevall M. \& Prat N. 2006b. Benthic macroinvertebrate assemblages and macrohabitat connectivity in Mediterranean-climate steams of northern California. Journal of North American Benthological Society. 25: 32-43.

Bonada N., Dolédec S. \& Statzner B. 2007. Taxonomic and biological trait differences of stream macroinvertebrate communities between Mediterranean and temperate regions: implications for future climatic scenarios. Global Change Biology. 13: 1658-1671.

Butcher T.J., Stuwart P.M. \& Simon T.P. 2003. A Benthic Community Index for streams in the Northern Lakes and Forests Ecoregion. Ecological Indicators. 3: 181193.

Couceiro C.R., Hamada N., Luz S.L., Forsberg B.R. \& Pimentel T.P. 2007. Deforestation and sewage effects on aquatic macroinvertebrates in urban streams in Manaus, Amazonas, Brazil. Hidrobiologia. 575: 271284.

Daufresne M. \& Boët P. 2007. Climate change on structure and diversity on fish communities in rivers. Global Change Biology. 13: 2467-2478.

DeShon J.E. 1995. Development and application of the invertebrate community index (ICI). In: Davis W.S., Simon T.P.(eds). Biological Assessment and Criteria: Tools for Water Resource Planning and Decision Making. pp. 217-243. CRC Press, Boca Raton, FL, 
U.S.A.

Durance I. \& Ormerod J. 2007. Climate change effects on upland stream macroinvertebrates over a 25-year period. Global Change Biology. 13: 942-957.

Fernandez H.R. \& Domínguez E. 2001. Guía para la determinación de artrópodos sudamericanos. Editorial Universitaria de Tucumán, Argentina.

Figueroa R., Valdovinos C., Araya E. \& Parra O. 2003. Macroinvertebrados bentónicos como indicadores de la calidad de agua de ríos del sur de Chile. Revista Chilena de Historia Natural. 76: 275-285.

Francou B. \& Pouyaud B. 2008. Glaciares: ¿Cómo y Dónde estudiarlos? Revista virtual Redesma. 2: 9-17.

Grim N.B., Chacón A., Dahm C.N, Hostetler S.W., Lind O.T., Starkweather P.L. \& Wurtsvaugh W.W. 1997. Sensitivity of aquatic ecosystems to climatic and anthropogenic changes: the basin and range, American Southwest and Mexico. Hydrological Processes. 11: 1021-1041.

Heino J., Muotka T. \& Paavola R. 2003. Determinants of macroinvertebrate diversity in headwater streams: regional and local influences. Journal of Animal Ecology. 72: 425-434.

Internationale Weiterbildung und Entwicklung gGmbH (InWEnt). 2008. Cambio Climático y retroceso de los glaciares en la zona Andina: Consecuencias para la Gestión de Recursos Hídricos. Revista virtual Redesma. 2: 19-23.

IPCC. 2007. Cambio climático 2007: Informe de síntesis. Contribución de los Grupos de trabajo I, II y III al Cuarto Informe de evaluación del Grupo Intergubernamental de Expertos sobre el Cambio Climático [Equipo de redacción principal: Pachauri, R.K. y Reisinger, A. (directores de la publicación)]. IPCC, Ginebra, Suiza.

Karr J.R. \& Chu E.W. 1999. Restoring life in running waters: Better Biological monitoring. Island Press. Washington, DC, U.S.A
Lake P.S. 2000. Disturbance, patchness and diversity in streams. Journal of the North American Benthological Society. 19: 573-592.

Merrit R. \& Cummins K. 1996. Acuatic Insect of North America. Second edition. Kendall y Hunt Publishing Company.

Metcalfe J.L. 1994. Biological Water-Quality Assessment of Rivers: Use of Macroinvertebrate Communities. In: Peter C., Jeoffrey E.P. (eds). The River Handbook: Hidrological and Ecological principles, pp. 145-170. Blackwell Scientific Publications press, Oxford.

Molina C.I. \& Gibon F.M. 2009. A new species of Cailloma Ross \& King, 1952 from Bolivia (Trichoptera, Hydrobiosidae). Revue française d'Entomologie (N.S.). 1: 23-29.

Moya N., Tomanova S. \& Oberdorff T. 2007. Initial development of a multi-metric index based on macroinvertebrates to assess streams condition in the Upper Isiboro-Sécure Basin, Cochabamba, Bolivia. Hidrobiología. 589: 107-116.

Navarro G. \& Maldonado M. 2002. Geografía Ecológica de Bolivia: Vegetación y ambientes acuáticos. Editorial: Centro de Ecología Difusión Simón I. Patiño. Bolivia.

Silveira M.P., Baptista B.F., Buss D.F., Nessimian J.L. \& Egler M. 2005. Application of biological measures for stream integrity assessment in south-east Brazil. Environmental Monitoring Assessment. 101: 117-128.

Tomanova S., Moya N. \& Oberdorff T. 2008. Using macroinvertebrate biological traits for assessing biotic integrity of Neotropical streams. River Research and Applications. 24: 1230-1239.

Vinson M.R. \& Hawkins C.P. 1998. Biodiversity of stream insects: variation at local, basin and regional scales. Annual Review of Entomology. 43: 271-293.

Vuille M. B., Francou P., Wagnon P., Juen I., Kaser G., Mark B.G. \& Bradley R. 2008. Climate change and tropical Andean glaciers: Past, present and future. Earth Science Reviews. 89: 79-96.

1 Unidad de Limnología y Recursos Acuáticos, Universidad Mayor de San Simón, CP 2352, Cochabamba, Bolivia,nabor.moya@gmail.

2 Institut de Recherche pour le Developpement UMR BOREA, Muséum National d'Histoire Naturelle, 43 rue Cuvier FR-75231 Paris, Francia.

3 Institut de Recherche pour le Developpement UMR BOREA, Muséum National d'Histoire Naturelle, 43 rue Cuvier FR-75231 Paris, Francia, francois-marie.gibon@ird.fr oberdorf@mnhn.fr.

4 Unidad de Limnología, Instituto de Ecología, Universidad Mayor de San Andrés, La Paz, Bolivia, laccodytes@yahoo.com.mx.

5 Instituto superior de Entomología, Instituto Miguel Lillo, Universidad Nacional de Tucumán, Argentina, mayfly@unt.edu.ar. 